\title{
Fluoreszenz-Bioimaging
}

\section{Fluorimetrische Bestimmung von intrazellulären Polyhydroxyalkanoaten}

CAROLA GRIEHL $^{1}$, ALEXANDER KETTNER ${ }^{1}$, MATTHIAS NOLL ${ }^{2}$, SIMONE BIELER ${ }^{1}$

${ }^{1}$ KOMPETENZZENTRUM ALGENBIOTECHNOLOGIE, HOCHSCHULE ANHALT, KÖTHEN

${ }^{2}$ INSTITUT FÜR BIOANALYTIK, HOCHSCHULE FÜR ANGEWANDTE WISSENSCHAFTEN, COBURG

\section{Polyhydroxyalkanoates (PHA) are synthesized as intracellular carbon} and energy storage compounds by many bacteria and are accumulated as granules in the cytoplasm of cells. Quantification of enriched PHA is of great importance for industrial production processes. Fluorescence measurements are particularly suitable for rapid determination of PHA during fermentation. With LipidGreen2 as fluorescent marker a fast and reliable assay for PHA monitoring in bioreactors was developed.

DOI: $10.1007 / \mathrm{s} 12268-021-1558-8$

(C) Die Autoren 2021

Vor dem Hintergrund des Klimawandels und einer notwendigen Reduktion persistenter petrochemischer Kunststoffe erobern biologisch abbaubare Kunststoffe auf Basis nachhaltiger Rohstoffe zunehmend den Markt. Bei einer global produzierten Kunst- stoffmenge von etwa 368 Millionen Tonnen ist der Marktanteil der Biokunststoffe mit 2,11 Millionen Tonnen noch sehr gering [1]. Aufgrund technischer Fortschritte bei den Herstellungsverfahren wird in den nächsten fünf Jahren ein Anstieg der Biokunststoffpro- duktion auf 2,87 Millionen Tonnen (2025) erwartet. Unter den Biokunststoffen gelten die biotechnologisch herstellbaren Polyhydroxyalkanoate (PHA) als schlafende Riesen, da sie biokompatibel sind und ähnliche Materialeigenschaften wie die im Kunststoffsektor vielfach genutzten, biologisch nicht abbaubaren, Polyolefine (PE, PP) besitzen.

Polyhydroxyalkanoate - biologisch abbaubare Biopolymere

PHA sind natürlich vorkommende Polyester aus D-Hydroxyalkansäuren $\left(\mathrm{C}_{3}\right.$ bis $\left.\mathrm{C}_{18}\right)$, die von vielen heterotroph und phototroph wachsenden Bakterien (wie Proteo- und Cyanobakterien) als Speicherstoffe für Kohlenstoff und Energie gebildet werden. Sie reichern sich in Form cytoplasmatischer Granula an, wenn Stressbedingungen wie N- oder P-Mangel vorliegen, und Kohlenstoff im Überschuss auftritt [2]. Ähnlich wie Triacylglyceride (TAG), die vor allem von Actinomyceten, Hefen, Pilzen, Mikroalgen und Pflanzen als Speicher-

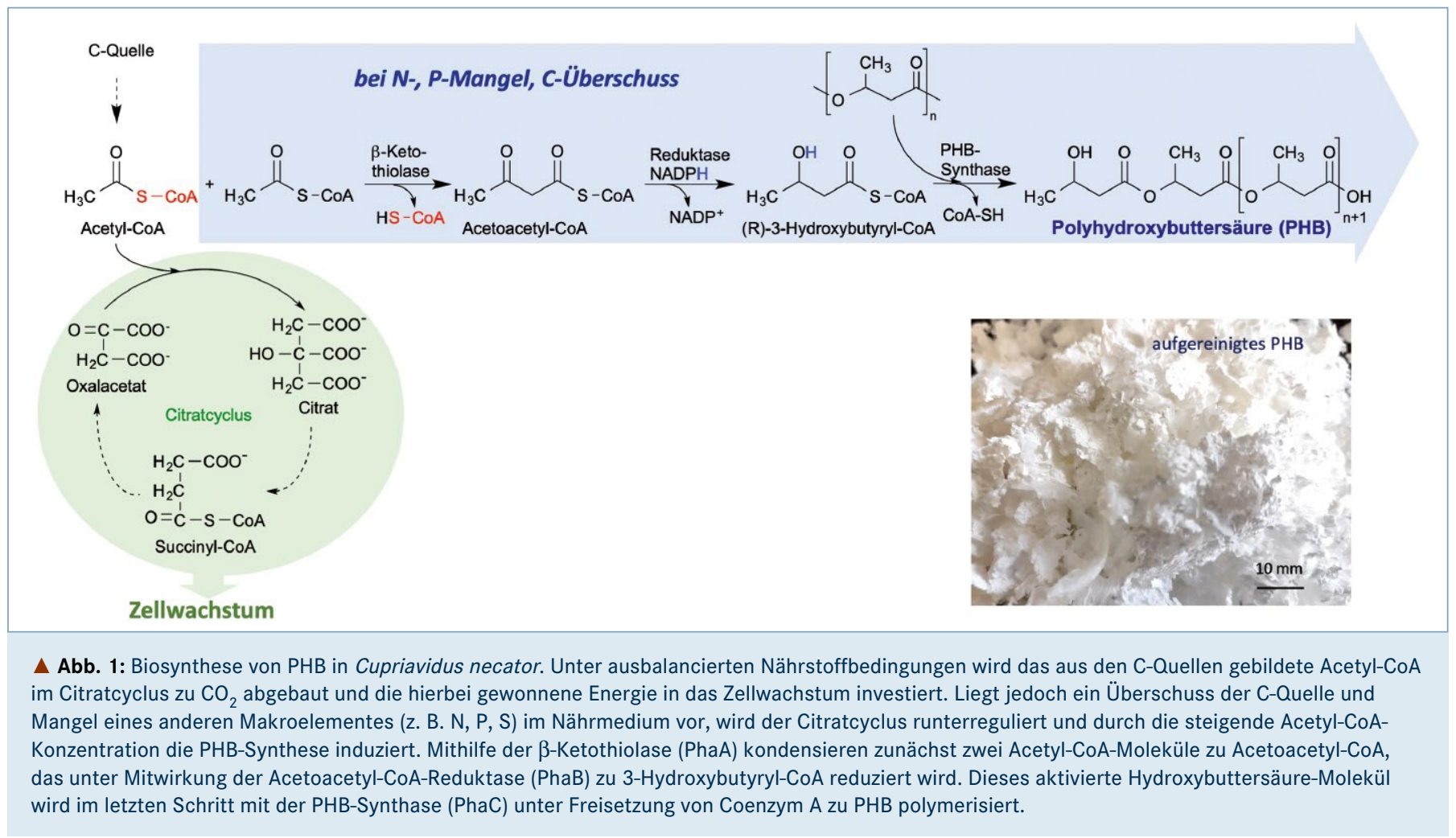


Tab. 1: Häufig gebildete PHA-Homopolyester und Copolyester.

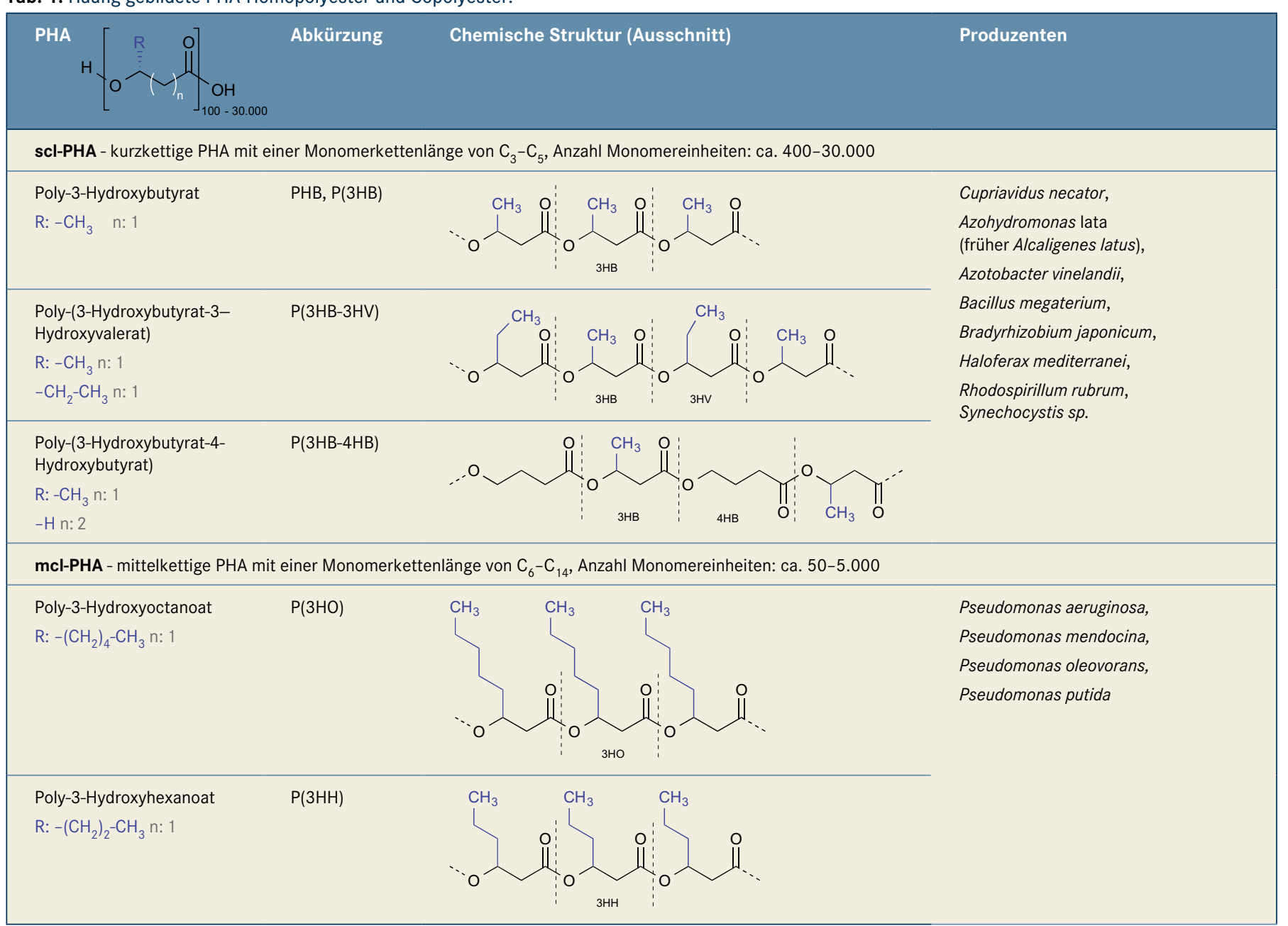

lipide gebildet werden, können PHA von den Zellen als Reserve mobilisiert werden, wenn eine Kohlenstoff-Mangelsituation auftritt.

Neben Homopolyestern, wie Poly-3-Hydroxybutyrat (PHB), synthetisieren Bakterien auch Heteropolymere (Tab. 1). Je nach Spezies und Substrat können Kettenlänge und Zusammensetzung sowie die Anzahl der Monomereinheiten variieren, was die mechanischen und thermischen Produkteigenschaften beeinflusst und vielfältige Anwendungen insbesondere im Verpackungsbereich (als PE- und PP-Ersatz) und medizinischen Bereich (resorbierbare Naht-/Stützmaterialien etc.) ermöglicht.

Hauptvertreter der PHA ist PHB, welches 1925 von Maurice Lemoigne als Zellinhaltsstoff in Bacillus megaterium entdeckt wurde [3] und in vielen Bakterien vorkommt. Besonders hohe Mengen an PHB (bis zu 95 \% der Biomasse) kann das aerobe Bodenbakterium Cupriavidus necator akkumulieren [2]. Cupriavidus necator ist ein stäbchenförmiges, gramnegatives Bakterium mit einer Größe von 0,7-1,3 $\mu \mathrm{m}$, das 1961 von Hans Günther Schlegel in der Nähe von Göttingen isoliert wurde [4]. Das Bakterium kann einerseits unter Verwertung organischer C-Quellen heterotroph wachsen, ist aber auch in der Lage, $\mathrm{CO}_{2}$ als anorganische Kohlenstoffquelle chemolithoautotroph zu metabolisieren. Bisher wurde angenommen, dass der Wildtyp C. necator H16 nur Gluconat und Fructose als Monosaccharide über den Entner-DoudoroffWeg verwerten kann, da das Schlüsselenzym der Glykolyse - die Phosphofructokinase fehlt. Der Arbeitskreis von Achim Kienle zeigte, dass auch glucosehaltige Medien nach einer kurzen Anpassungszeit einen glucosepositiven Phänotyp in C. necator H16 induzieren [5], sodass Glucose als preiswerte C-Quelle nicht nur von Cupriavidus-Mutanten verwertet werden kann. Die enzymkatalysierte PHB-Biosynthese (Abb. 1) wird unter Nährstofflimitation bei gleichzeitigem C-Überschuss induziert. Unter diesen Bedingungen liegt Acetyl-CoA im Überschuss vor und wird über Hydroxyacyl-CoA zum Polymer umgewandelt und intrazellulär in nativen Granula eingeschlossen. Der Wildtyp H16 kann unter geeigneten Kultivierungsbedingungen bis zu einem Dutzend PHB-Granula mit einer Größe von 200-500 nm im Cytoplasma anreichern [6].

\section{Fluoreszenzfarbstoffe zur Quantifizierung von PHA}

Für die Entwicklung effizienter biotechnologischer Herstellungsverfahren von PHA ist eine schnelle Quantifizierung des Produkts unerlässlich. Häufig genutzte chromatographische, spektroskopische und gravimetrische Methoden zur Bestimmung von PHA sind meist zeit- und materialaufwendig, da die Polymere vor der Analyse erst aus den Zellen extrahiert werden müssen.

Fluoreszenzbasierte Assays ermöglichen eine schnelle Analyse mikrobieller Zellinhaltsstoffe während des Fermentationsprozesses. Grundlage hierfür ist, dass die Fluoreszenzfarbstoffe die Zellhüllen passieren und selektiv an die Biomoleküle binden können. Nach Lichtanregung $\left(\lambda_{E x}\right)$ erzeugen die Biomolekül-Farbstoffkomplexe ein Fluoreszenzsignal, das zur quantitativen Bestimmung dieser Stoffe genutzt werden kann. Speicherlipide, wie PHA, können durch geeignete hydrophobe Farbstoffmoleküle angefärbt und über die Intensität der detektierten Fluoreszenzemission quantifiziert werden. In Lösungen mit einer Zellkonzentration bis 0,1 (OD) ist das Fluoreszenzsignal 


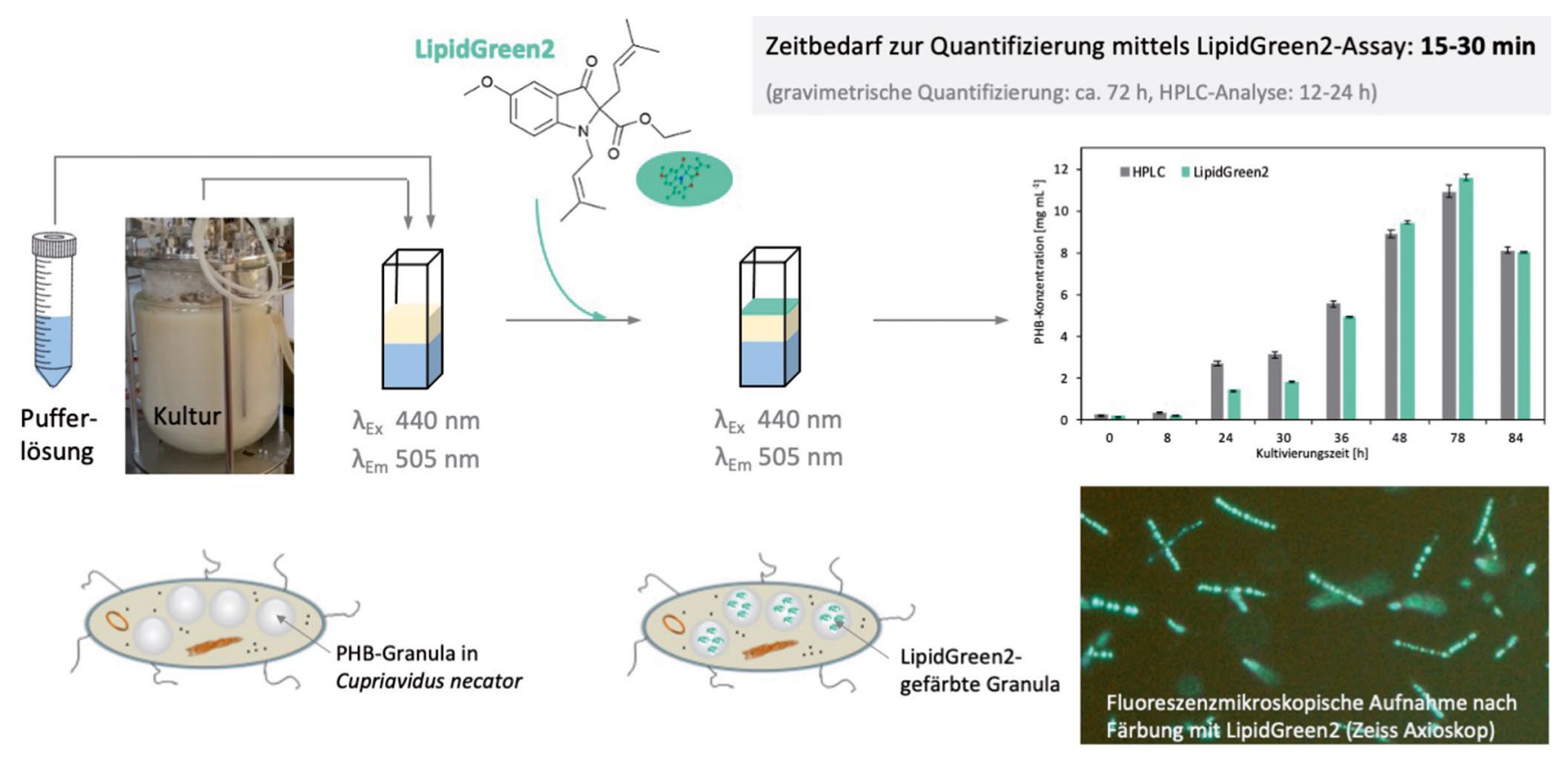

Abb. 2: Methode zur schnellen Quantifizierung von PHB in Cupriavidus necator mittels LipidGreen2-Bioassay: 1,5 ml PBS-Puffer werden mit 0,5 ml der Kultursuspension gemischt und das Seitwärtsstreulicht bei $\lambda_{\mathrm{Ex}} 440 \mathrm{~nm} / \lambda_{\mathrm{Em}} 505 \mathrm{~nm}$ aufgezeichnet. Anschließend erfolgt die Zugabe von $100 \mu \mathrm{l}$ LipidGreen2-DMSO-Lösung. Nach 10 min Inkubation wird die Fluoreszenz bei $\lambda_{\mathrm{Ex}} 440 \mathrm{~nm} / \lambda_{\mathrm{Em}} 505 \mathrm{~nm}$ gemessen. Der Einfluss der Zellen/Biomasse (Inner-Filter-Effekte) wird durch Verrechnung des Fluoreszenzsignals mit dem Seitwärtsstreulicht korrigiert [12].

proportional zur Konzentration des FarbstoffPHA-Komplexes.

Besonders häufig wurden bisher die Fluoreszenzfarbstoffe Nilrot und BODIPY eingesetzt [7-9]. BODIPY-Farbstoffe zeichnen sich durch eine scharfe Emissionsbande und hohe Quantenausbeuten aus. Allerdings wird die Quantifizierung durch eine hohe Hintergrundfluoreszenz erschwert. Der lipophile rote Phenoxazonfarbstoff Nilrot zeigt hingegen eine geringe Hintergrundfluoreszenz und wird daher häufig zum Anfärben hydrophober Zellbestandteile, wie Lipiden, eingesetzt. Allerdings ist eine Unterscheidung zwischen unpolaren Speicherlipiden und polareren Membranlipiden schwierig, da Nilrot an alle Lipidmoleküle bindet und sich diese in ihren fluoreszenzspektroskopischen Eigenschaften kaum unterscheiden. Aufgrund der geringen Sensitivität und der schnell nachlassenden Intensität der Färbung sowie der bei höheren Konzentrationen beobachteten Quenching-Effekte ist eine reproduzierbare und genaue Quantifizierung mit Nilrot häufig nicht zuverlässig möglich.

LipidGreen, ein neu entwickelter lipophiler Fluoreszenzfarbstoff mit einem IndolinGrundgerüst, zeichnet sich durch eine höhere und langzeitstabilere Fluoreszenz als Nilrot aus und färbt mit hoher Selektivität Neutrallipide, wie am Beispiel von PHB-produzierenden Mikroorganismen gezeigt werden konnte [10].

Mit dem weiterentwickelten Farbstoff LipidGreen2, der 2013 erstmals durch H. S.
Chun et al. für die mikroskopische Färbung von Neutrallipiden in Zellen und Fettdepots des Zebrafisches [11] beschrieben wurde, konnte die Fluoreszenzeffizienz noch verbessert werden. LipidGreen2 bewirkte im Vergleich zu Nilrot eine stärkere Färbung der Lipideinschlüsse bei minimalem nicht spezifischem Hintergrundrauschen.

Der cLogP-Wert, der ein Modellmaß für das Verhältnis zwischen Lipophilie (Fettlöslichkeit) und Hydrophilie (Wasserlöslichkeit) einer Substanz ist, gibt Auskunft über die Hydrophobizität. Für LipidGreen2 liegt dieser Wert mit 5,92 deutlich höher als für Nilrot $(4,62)$, was die höhere Selektivität von LipidGreen2 für Neutrallipide erklärt (Tab. 2).

\section{LipidGreen2-Bioassay - Quantifizierung von PHB in Cupriavidus necator}

Vergleichende Untersuchungen von Nilrot und LipidGreen2 zur Bestimmung von Lipiden in PHB-angereicherten CupriavidusStämmen und Negativmutanten bestätigten die höhere Selektivität von LipidGreen2 für Speicherlipide. Während bei Färbung mit Nilrot die Fluoreszenzintensität für PHB nur geringfügig höher war als für Membranlipide, konnte für LipidGreen2 ein etwa 4fach höheres Signal für PHB detektiert werden. Fluoreszenzmikroskopische Beobachtungen bestätigten die hohe Affinität von LipidGreen2 für Neutrallipide. Auf Basis dieser und weiterer Untersuchungen zur Zellwand- passage beider Farbstoffe (unveröffentlichte Ergebnisse) wurde ein fluoreszenzbasierter Assay entwickelt, der eine schnelle und einfach durchführbare Online-Überwachung von PHA während der biotechnologischen Kultivierung von PHA-produzierenden Mikroorganismen gestattet [12]. Um eine zuverlässige Quantifizierungsmethode mit möglichst geringem Zeitbedarf zu etablieren, wurde der Einfluss biotischer und abiotischer Faktoren auf das Fluoreszenzverhalten untersucht und die Methode angepasst. Mit dem LipidGreen2-basierten Bioassay gelang das Bioimaging von intrazellulären PHBGranula am Modellorganismus C. necator. Die erhaltene Linearität bei unterschiedlichen Biomassekonzentrationen der Kultursuspension $\left(R^{2}>0,98\right.$ bis $\left.10 g_{T S} / L\right)$ gestattet eine genaue PHB-Quantifizierung in weniger als 30 Minuten nach einem einfach durchführbaren Protokoll (Abb. 2). Neben einer hohen Fluorophorstabilität zeichnet sich der Asssay durch eine hohe und reproduzierbare Nachweisgenauigkeit bei geringem Hintergrundrauschen sowie durch eine hohe Sensitivität für den Nachweis lipophiler Speicherstoffe aus. Um die Übertragbarkeit auf andere Spezies und Geräte zu gewährleisten, wurde ein Standardisierungsverfahren des Fluoreszenzsignals entwickelt und die Ergebnisse mit chromatographischen Analysen verglichen.

LipidGreen2 erwies sich als ein vielversprechender, einfach zu handhabender Fluoreszenzmarker für die selektive Bestimmung 
Tab. 2: Eigenschaften von LipidGreen und LipidGreen2 im Vergleich zu Nilrot.

\begin{tabular}{|c|c|c|c|}
\hline & LipidGreen & LipidGreen2 & Nilrot \\
\hline \multicolumn{4}{|l|}{ Struktur } \\
\hline Summenformel & $\mathrm{C}_{18} \mathrm{H}_{21} \mathrm{NO}_{4}$ & $\mathrm{C}_{22} \mathrm{H}_{29} \mathrm{NO}_{4}$ & $\mathrm{C}_{20} \mathrm{H}_{18} \mathrm{~N}_{2} \mathrm{O}_{2}$ \\
\hline Mol. Masse $[\mathrm{g} / \mathrm{mol}]$ & 315,4 & 371,2 & 318,1 \\
\hline Zielstrukturen & Neutrallipide & Neutrallipide & Neutral- und Polarlipide \\
\hline Messbereich $\lambda_{\mathrm{Ex}} / \lambda_{\mathrm{Em}}[\mathrm{nm}]$ & $450 / 510$ & $440 / 500-510$ & $480-530 / 575-620$ \\
\hline $\begin{array}{l}\text { Hydrophobizität clogP } \\
\text { (kalkuliert mit ChemDraw Ultra 11.0) }\end{array}$ & 4,07 & 5,92 & 4,62 \\
\hline
\end{tabular}

von Speicherlipiden in Mikroorganismen. Die für die Bestimmung von PHB in C. necator etablierte Methode wird gegenwärtig auf weitere PHA- und TAG-produzierende Mikroorganismen übertragen.

Lesen Sie mehr zu Bioplastik-produzierenden Bakterien im Artikel von Moritz Koch und Karl Forchhammer auf S. 200-201.

\section{Literatur}

[1] European Bioplastics (2020) Bioplastics market data. https://www.european-bioplastics.org/market/ (letzter Zugriff: 09. Februar 2021)

[2] Steinbüchel A, Oppermann-Sanio F, Ewering C, Pötter M (2013) Versuche. In: Mikrobiologisches Praktikum. SpringerLehrbuch. Springer Spektrum, Berlin, Heidelberg [3] Lemoigne M (1926) Produits de déshydration et de polymérisation de l'acide $\beta$-oxobutyrique. Bull Soc Chem Biol (Paris) 8: 770

[4] Schlegel HG, Gottschalk G, von Bartha R (1961) Formation and utilization of PHB knallgas bacteria (Hydrogenomonas). Nature 191: 463-465

[5] Franz A, Rehner R, Kienle A et al. (2011) Rapid selection of glucose-utilizing variants of the polyhydroxyalkanoate producer Ralstonia eutropha $\mathrm{H} 16$ by incubation with high substrate levels. Lett Appl Microbiol 54: 45-51

[6] Wieczorek R, Pries A, Steinbüchel A et al. (1995) Analysis of a 24-kilodalton protein associated with the polyhydroxyalkanoic acid granules in Alcaligenes eutrophus. J Bacteriol 9: 2425-2435

[7] Gorenflo V, Steinbüchel A, Marose S et al. (1999) Quantification of bacterial polyhydroxyalkanoic acids by Nile red staining. Appl Microbiol Biotechnol 51: 765-772 [8] Elain A, Le Fellic M, Corre YM et al. (2015) Rapid and qualitative fluorescence-based method for the assessment of PHA production in marine bacteria during batch culture. World J Microbiol Biotechnol 31: 1555-1563

[9] Karmann S, Follonier S, Bassas-Galia M et al. (2016) Robust at-line quantification of poly(3-hydroxyalkanoate) biosynthesis by flow cytometry using a BODIPY 493/503-SYTO 62 double-staining. J Microbiol Methods 131:166-171 [10] Choi JE, Na HY, Yang TH et al. (2015) A lipophilic fluorescent LipidGreen1-based quantification method for high-throughput screening analysis of intracellular poly-3hydroxybutyrate. AMB Express 5: 131

[11] Chun HS, Jeon JH, Pagire HS et al. (2013) Synthesis of LipidGreen2 and its application in lipid and fatty liver imaging. Mol Biosyst 9: 630-633

[12] Kettner A, Griehl C (2020) The use of LipidGreen2 for accurate visualization and quantification of intracellular polyhydroxybutyrate in Cupriavidus necator. Biochem Biophys Rep 24: 100819

Funding note: Open Access funding enabled and organized by Projekt DEAL. Open Access: Dieser Artikel wird unter der Creative Commons Namensnennu B.O International Lizenz veröffentlicht, welche die Nutzung, Vervielfältigung, erlaubt, sofern Sie den/die ursprünglichen Autor(en) und die Quelle ordnungsgemäß nennen, einen Link zur Creative Commons Lizenz beifügen und angeben, ob Änderungen vorgenommen wurden. Die in diesem Artikel enthaltenen Bilder und sonstiges Drittmaterial unterliegen ebenfalls der genannten Creative Commons Lizenz, sofern sich aus der Abbildungslegend nichts anderes ergibt. Sofern das betreffende Material nicht unter der genannten Creative Commons Lizenz steht und die betreffende Handlung nich Weiterverwendungen des Materials die Einwilligung oben aufgefüh Weiterverwendungen des Materials die Einwilligung des jeweiligen
Rechteinhabers einzuholen. Weitere Details zur Lizenz entnehmen Sie bitte der Rechteinhabers einzuholen. Weitere Details zur Lizenz entnehmen Sie bitte der

Korrespondenzadresse:

Prof. Dr. Carola Grieh

Kompetenzzentrum Algenbiotechnologie Hochschule Anhalt

Bernburger Straße 55 D-06366 Köthen

carola.griehl@hs-anhalt.de

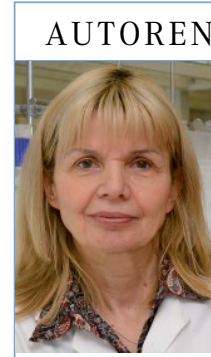

\section{Carola Grieh}

Chemiestudium und Promotion an der Universität Halle. Seit 1997 Professorin für Biochemie und Phototrophe Biotechnologie, Hochschule Anhalt. Seit 2000 Leiterin des Kompetenzzentrums Algenbiotechnologie. 2011 Mitbegründerin des Biosolarzentrums, Hochschule Anhalt \& GICON GmbH. 2019 Gründerin des Zentrums Naturstoffbasierte Therapeutika (ZNT) in Kooperation mit Fraunhofer-IZI-MWT. Seit 2020 Direktorin des Center of Life Sciences der Hochschule Anhalt.

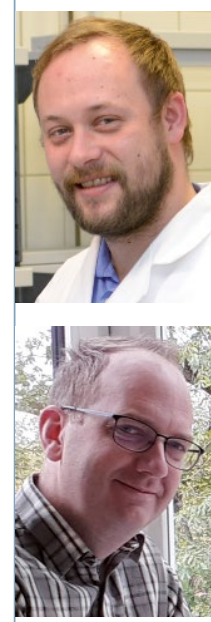

\section{Alexander Kettner}

Studium der Biochemie an der Universität Greifswald mit Schwerpunkt molekulare Biotechnologie/Proteinengineering. 2015-2016 Gastwissenschaftler an der EGE Universität (Izmir, Türkei) zum Thema Thermostabile Cellulasen. Seit 2016 Wissenschaftlicher Mitarbeiter und Doktorand (in Kooperation mit HS Coburg/Universität Bayreuth) im Kompetenzzentrum Algenbiotechnologie an der Hochschule Anhalt.

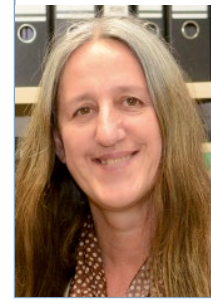

Matthias Noll

Biologiestudium an der Universität Kassel und Promotion an der Universität Marburg. 2004-2006 Postdoc, Max-Planck.Institut für terrestrische Mikrobiologie, Marburg, und ETH, Zürich. 2012 Habilitation an der TU Berlin. Seit 2012 Professor an der Hochschule für angewandte Wissenschaften Coburg. Seit 2014 Privatdozent an der Universität Bayreuth. 2017 Gründer des Institutes für Bioanalytik in Coburg.

\section{Simone Bieler}

Chemiestudium an der Universität Halle. 1986-1991 Mitarbeiterin F\&E VEB Chemische Werke Buna. 1992-1997 Wissenschaftliche Mitarbeiterin am Institut für Organische Chemie der Universität Halle-Wittenberg. Seit 1997 Wissenschaftliche Mitarbeiterin im Kompetenzzentrum Algenbiotechnologie an der Hochschule Anhalt. Seit 2004 Lehrkraft für besondere Aufgaben im Bereich Biochemie an der Hochschule Anhalt. 